Received: 2017.02.12

Accepted: 2017.03 .10

Published: 2017.12.15

\title{
The Predictive Value of Multidetector High Resolution Computed Tomography in Evaluation of Suspected Sputum Smear Negative Active Pulmonary Tuberculosis in Egyptian Zagazig University Hospital Patients
}

Authors' Contribution:
A Study Design
B Data Collection
C Statistical Analysis
D Data Interpretation
E Manuscript Preparation
F Literature Search
G Funds Collection

\author{
Ahmed Mohamed Alsowey ${ }^{1 \mathrm{ADF}}$, Mohamed Ibrahim Amin ${ }^{1 \mathrm{CE}}$, Ahmed Mohamed Said ${ }^{2 \mathrm{BG}}$ \\ ${ }^{1}$ Department of Radiodiagnosis, Faculty of Medicine, Zagazig University, Zagazig, Egypt \\ 2 Department of Chest, Faculty of Medicine, Zagazig University, Zagazig, Egypt
}

Author's address: Ahmed Mohamed Alsowey, Department of Radiodiagnosis, Faculty of Medicine, Zagazig University, Zagazig, Egypt, e-mail: ahmedalsowey@yahoo.com

\begin{abstract}
Summary
The aim of this work is to study the usage of multi-detector HRCT chest in diagnosing pulmonary TB cases whose sputum smears are negative and making a correlation between their CT features and their sputum culture results.

This study was carried out from December 2014 to December 2016 at Zagazig university hospitals, Radiodiagnosis department. It included 150 patients. Their ages ranged between 10 to 70 years with a mean age 40 years. They were referred from the outpatient respiratory medicine clinic of the hospital. All patients had been presented clinically with suspicion of PTB. Their clinical features and HRCT findings were investigated to predict the risk for PTB. We then designed provisional HRCT diagnostic criteria based on the results to rank the risk of PTB.

A positive tuberculin skin test alone among clinical laboratory findings was significantly associated with an increase of risk of PTB. Centrilobular nodules, large nodules, tree-in-bud appearance and the main lesion being located in S1, S2, and S6 lung segments were significantly associated with an increased risk of PTB. At HRCT 40 out of 44 patients with class III ranking showed active pulmonary TB. 12 out of 51 patients presented with class II ranking and 8 out of 40 were ranked as class I. The sensitivity, specificity and positive likelihood ratio of class I ranking HRCT criteria to diagnose active pulmonary TB were $95 \%, 40 \%$ and 1.4, respectively. Class II ranking results were $85 \%, 72 \%, 3$, respectively. Finally class III ranking results were $45 \%, 90 \%, 11.5$, respectively.
\end{abstract}

Cases suspected of having active pulmonary TB whose smears are negative can benefit from MD HRCT chest findings to predict those patients of high risk with good reproducibility.

MeSH Keywords: $\quad$ Multidetector Computed Tomography • Pulmonary Tuberculosis • Sputum Culture

PDF file: $\quad$ http://www.polradiol.com/abstract/index/idArt/903743

\section{Background}

Tuberculosis (TB) is one of the infectious disease worldwide, being one of the commonest causes of death, after Human Immunodeficiency Virus (HIV) infection, with an estimated 8.7 million new cases of TB in 2011 [1].

Nearly one-half to one-third of the pulmonary tuberculosis (PTB) cases are smear-negative and approximately 1.9 million new PTB cases were found to be smear-negative in $2011[2]$.

The sensitivity of sputum smear examination for acid-fast bacilli (AFB) is poor, therefore, rapid and accurate diagnosis of smear negative pulmonary tuberculosis (TB) is warranted. A high probability of PTB needs to be identified among sputum smear-negative findings without missing patients who do indeed have PTB [3]. A significant proportion of the 
patients treated for smear negative PTB never have bacteriological confirmation, even with improved culture methods, therefore, most cases of smear-negative PTB are diagnosed on the basis of the clinical presentation, radiological findings and other laboratory tests [1].

In cases of PTB, the presence of cavities and satellite lesions detected at computed tomography (CT) scans and cannot be detected at chest X-ray, can provide more accurate information about the extent and distribution of the disease process. Furthermore, CT can distinguish cases with active from old infection process [4].

It had been shown that presence of cavities and airspace consolidation could be related to the degree of positivity in PTB patients' smears. There are some pieces of data about the relationship between morphologic findings on high-resolution computed tomography (HRCT) and the number of AFB on sputum smears in patients with PTB $[5,6]$.

The aim of this work is to study the usage of multi-detector HRCT chest in diagnosing pulmonary TB cases whose sputum smears are negative and making a correlation between their CT features and their sputum culture results.

\section{Material and Methods}

\section{Patients}

A total of 150 Egyptian patients were included in the study, that had been conducted from December 2014 to December 2016 at multidetector CT unit, Zagazig University hospitals.

Patients were referred from the respiratory medicine outpatient clinic, Zagazig University hospital. Their ages range from 10 to 70 years old with a mean of 40 years.

Patients included are those with high suspicion of having active pulmonary TB (By clinical examination and $\mathrm{X}$ ray findings) and showing smear negative for acid-fast bacilli (AFB) in three consecutive sputum samples.

Those having positive AFB in their sputum smears were excluded. HIV or immune-compromised patients were not included in this study as well. Those with atypical MD-HRCT findings for PTB were followed up clinically and even biopsied.

All participants signed a written informed consent and filled a written survey including demographic and clinical data. The protocol and informed consent forms used in this study were approved by the Institutional Review Board (IRB) of Zagazig University.

\section{Methods}

All patients were subjected to:

1. History taking: must exclude any chronic chest disease, old TB and those taking anti-tuberculous treatments.

2. Symptom analysis: any symptoms of pulmonary TB such as: cough, hemoptysis, loss of weight, fever or night sweating.

3. Clinical examination.
Table 1. MD-HRCT findings in patients with sputum negative pulmonary TB.

\begin{tabular}{lrc}
\hline \multicolumn{1}{c}{ MD- HRCT findings } & \multicolumn{2}{c}{ Number of cases (\%) } \\
\hline Centri-lobular nodules & 135 & $(90 \%)$ \\
\hline Large nodules & 90 & $(60 \%)$ \\
\hline Tree-in-bud & 50 & $(33.33 \%)$ \\
\hline Peribronchial thickening & 29 & $(19.33 \%)$ \\
\hline Traction bronchiectasis & 24 & $(16 \%)$ \\
\hline Lymph node enlargement & 23 & $(15.33 \%)$ \\
\hline Consolidation & 20 & $(13.33 \%)$ \\
\hline Calcified granuloma & 19 & $(12.66 \%)$ \\
\hline Ground glass opacity & 17 & $(11.33 \%)$ \\
\hline Cavity & 12 & $(8 \%)$ \\
\hline Mass & 10 & $(6.66 \%)$ \\
\hline Emphysema & 5 & $(3.33 \%)$ \\
\hline NB - More than one finding could be seen in the same patient.
\end{tabular}

\section{Tuberculin skin test.}

5. Bacteriologic analysis: Three successive sputum samples for three successive days were collected in the early morning for detection of AFB. If the process of spontaneously giving sputum by the patient not possible, we used hypertonic saline nebulizer in the early morning preceded by salbutamol inhalation for induction of sputum. Direct smear examination of the samples were done after that using Zeihl-Neelsen stain. Culturing for mycobacterial tuberculosis was done using Lowenstein Jensen media. Finally the samples were incubated for 8 weeks before the final results collected.

6. HRCT chest: Through 128-row multidetector CT scanner (PHLIPS Ingenuity Core 128 TM, Philips Healthcare Nederland B.V., Veenpluis 4-6, 5684 PC Best, The Netherlands), the chest MDCT studies were performed utilizing 1.25 mm slice thickness, 120: $140 \mathrm{kV}$, 400: 600 mAs, $512 \times 512$ matrix size. Examination was done while the patient is lying supine during single breath-hold in a cranial-to-caudal direction. Using high spatial resolution algorithm [7], series of contiguous thin-collimation 1.25 $\mathrm{mm}$ axial high resolution images were reconstructed from the volumetric data set. Images were analyzed in both pulmonary and mediastinal window.

\section{Image analysis}

Obtained images were assessed for the existence of centrilobular nodules, tree in bud pattern (indicate endobronchial spread of infection), larger nodules, masses, lobular consolidations, cavities, bronchoceles, ground glass opacities and mediastinal lymph node enlargement (Table 1). The distribution of findings was assessed for the presence of a main lesion in lung segments namely S1, S2 and S6.

To predict the risk for active pulmonary TB, we applied the criteria designed by Nakanishi et al., [3] that were based on the combination of HRCT findings, subsequently the 
Table 2. Ranking of patients according to MD-HRCT findings.

\begin{tabular}{cll}
\hline Rank number & \multicolumn{1}{c}{ Description } & \multicolumn{1}{c}{ HRCT findings required for diagnosis } \\
\hline 3 (III) & Highly suspect pulmonary TB (PTB) & $\begin{array}{l}\text { Presence of at least three of the following findings: } \\
\text { 1.Main lesion in S1, S2 or S6 segments. } \\
\end{array}$ \\
& & $\begin{array}{l}\text { 2.Tree in bud appearance. } \\
\text { 3.Lobular consolidations. } \\
\text { 4.Larger nodules. }\end{array}$ \\
\hline 2 (II) & Probable pulmonary TB (PTB) & $\begin{array}{l}\text { Presence of at least two of the following findings: } \\
\text { 1.Main lesion in S1, S2 or S6 segments. }\end{array}$ \\
& & 2.Tree in bud appearance. \\
& & 3.Lobular consolidations. \\
\hline 1 (I) & 4.Larger nodules. \\
\hline 0 & Non-specific or difficult to differentiate from other disease & Lesions located mainly in the middle lobe or lingular segment. \\
\hline
\end{tabular}

Table 3. Demographic data of our patients.

\begin{tabular}{cccc}
\hline Age & Male & Female & Total \\
\hline $10:<20$ years & 6 & 2 & 8 \\
\hline $20:<30$ years & 14 & 7 & 21 \\
\hline $30:<40$ years & 44 & 21 & 65 \\
\hline $40:<50$ years & 20 & 10 & 30 \\
\hline $50:<60$ years & 15 & 2 & 17 \\
\hline $60: 70$ years & 6 & 3 & $150(100 \%)$ \\
\hline Total $($ No, $\%)$ & $105(70 \%)$ & $45 \%)$ & 9 \\
\hline
\end{tabular}

patients were ranked from 0 to 3 (Table 2). Two radiologists record each HRCT finding and decide the rank.

\section{Statistical analysis}

Data collected was revised, coded, tabulated and introduced to personal computer for statistical analysis. All data manipulation and analysis were performed using the $20^{\text {th }}$ version of SPSS (Statistical Package for Social Sciences). We applied stepwise regression, to determine the HRCT findings that had significant association with increased risk of pulmonary TB. Analysis of inter-observer variation for every finding was done using Kappa test (the $\kappa$ statistics). Using a weighted $\kappa$ statistics, the interobserver variation in the ranking process for every patient was examined.

\section{Results}

The mean age of the patients was 40 years. Age group between 30 and 40 years was the chiefly affected group (Table 3). Predominance of male gender $(70 \%$ of the patients) was noticed. Cough was the commonest symptom, presented in $90 \%$ of the patients, followed by chest pain in $66 \%$, expectoration in $53 \%$, hemoptysis in $30 \%$, fever in $13 \%$, weight loss in $8 \%$, night sweating in $6 \%$ and dyspnea in $4 \%$ of the patients (Table 4 ).
Table 4. The presenting symptoms of our patients.

\begin{tabular}{lrc}
\hline The presenting symptom & \multicolumn{3}{c}{ Number of cases (\%) } \\
\hline Cough & 136 & $(90.66 \%)$ \\
\hline Chest pain & 100 & $(66.66 \%)$ \\
\hline Expectoration & 80 & $(53.33 \%)$ \\
\hline Hemoptysis & 45 & $(30 \%)$ \\
\hline Fever & 20 & $(13.33 \%)$ \\
\hline Weight loss & 12 & $(8 \%)$ \\
\hline Night sweating & 9 & $(6 \%)$ \\
\hline Dyspnea & 6 & $(4 \%)$ \\
\hline
\end{tabular}

NB - More than one symptom could be present in the same patient.

The final diagnosis of pulmonary TB at that work was dependent on AFB culture results which considered a gold standard for diagnosing pulmonary tuberculosis.

The commonest HRCT findings were centrilobular nodules $(90 \%)$, large nodules $(60 \%)$ and tree in bud appearance (33\%). The complete radiological findings are presented in Table 1. 
Table 5. The lobar distribution of the lesions.

\begin{tabular}{lrc}
\hline The lung lobe & \multicolumn{2}{c}{ Number of cases (\%) } \\
\hline Right upper lobe & 90 & $(60 \%)$ \\
\hline Left upper lobe & 30 & $(20 \%)$ \\
\hline Right lower lobe & 15 & $(10 \%)$ \\
\hline Left lower lobe & 10 & $(6.67 \%)$ \\
\hline Right middle lobe & 5 & $(3.33 \%)$ \\
\hline
\end{tabular}

The localization of the radiological lesions was also evaluated. Radiological lesions were observed in the right $(60 \%)$ and left (20\%) upper lobes most frequently (Table 5).

Based on the ranking obtained by CT features, patients were divided into three groups:

1. Rank I: At HRCT only eight out of the 40 patients ranked as I had final diagnosis of active pulmonary TB while in the remaining patients five had non-tuberculous mycobacterium infection (NTM), 11 had bronchopneumonia, three had cryptogenic organizing pneumonia (COP) and two had allergic broncho-pulmonary aspergillosis (ABPA).
2. Rank II: Only 12 out of the 51 patients who were ranked as II had final diagnosis of active pulmonary TB, NTM were found in six patients, bronchopneumonia in seven patients, COP in six patients and ABPA in four patients.

3. Rank III: 40 out of 44 patients were ranked as III had final diagnosis of active pulmonary TB. NTM were found in two patients. One of the patients had final diagnosis of bronchopneumonia (Table 6).

4. Calculating the sensitivity, specificity and positive likelihood ratio of the different class ranking using HRCT criteria for diagnosing active pulmonary TB were as follow: Rank III was 45\%, 90\% and 11.5 , respectively, while in rank II it was 85\%, 72\%, 3, respectively and in rank I it was 95\%, 40\%, 1.4, respectively (Table 7). Figures 1-5 demonstrate some of the MD-HRCT features in Rank III patients.

\section{Discussion}

Pulmonary TB can mimic a lot of diseases in clinical and laboratory findings, reliability on sputum smear has many limitations as sputum may be false negative if the disease is mild, decreased load of bacilli in the sputum sample and patient is giving saliva instead of sputum. The sputum culture take several weeks for diagnosis of PTB. Patients with suspected pulmonary $\mathrm{TB}$ whose sputum smears are negative for $\mathrm{AFB}$

Table 6. The final diagnosis of the suspected sputum smear-negative PTB on MD-HRCT criteria.

\begin{tabular}{|c|c|c|c|c|c|}
\hline \multirow{2}{*}{ Final diagnosis } & \multicolumn{4}{|c|}{ HRCT criteria } & \multirow{2}{*}{ Tota } \\
\hline & Rank 0 & Rank I & Rank II & Rank III & \\
\hline Pulmonary TB & 0 & 8 & 12 & 40 & 60 \\
\hline NTM & 3 & 5 & 6 & 2 & 16 \\
\hline Bronchopneumonia & 1 & 11 & 7 & 1 & 20 \\
\hline COP & 1 & 3 & 6 & 0 & 10 \\
\hline ABPA & 1 & 2 & 4 & 0 & 7 \\
\hline Sarcoidosis & 3 & 2 & 5 & 1 & 11 \\
\hline Lung cancer & 1 & 2 & 1 & 0 & 4 \\
\hline Lymphoma & 1 & 3 & 1 & 0 & 5 \\
\hline Panbronchiolitis & 3 & 2 & 4 & 0 & 9 \\
\hline Septic emboli & 1 & 4 & 3 & 0 & 8 \\
\hline Total & 15 & 40 & 51 & 44 & 150 \\
\hline
\end{tabular}

NTM - nontuberculous mycobacterium; COP - cryptognic organizing pneumonia; ABPA - allergic bronchopulmonary aspergillosis.

Table 7. Sensitivity, specificity, positive and negative likelihood ratios for each rank of MD-HRCT diagnosis of sputum culture proved pulmonary TB cases.

\begin{tabular}{lccc}
\hline & Rank I & Rank II & Rank III \\
\hline Sensitivity & $95 \%$ & $85 \%$ & $45 \%$ \\
\hline Specificity & $40 \%$ & $72 \%$ & $90 \%$ \\
\hline Positive likelihood ratio & 1.4 & 3 & 11.5 \\
\hline Negative likelihood ratio & 0 & 0.3 & 0.5 \\
\hline
\end{tabular}




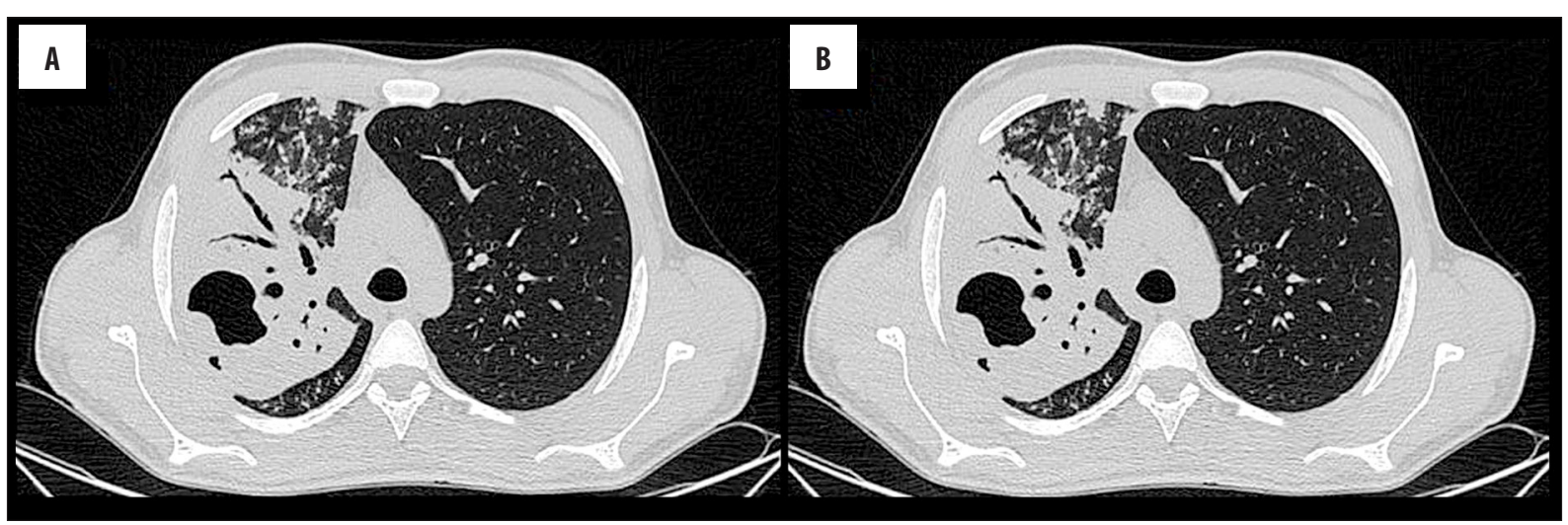

Figure 1. (A, B) Axial MD HRCT (pulmonary window) showing centrilobular nodules with tree in bud configuration, consolidated area with air bronchogram and cavity formation in the right upper lobe anterior segment in a 21 years old male complaining of low grade fever, cough and expectoration.

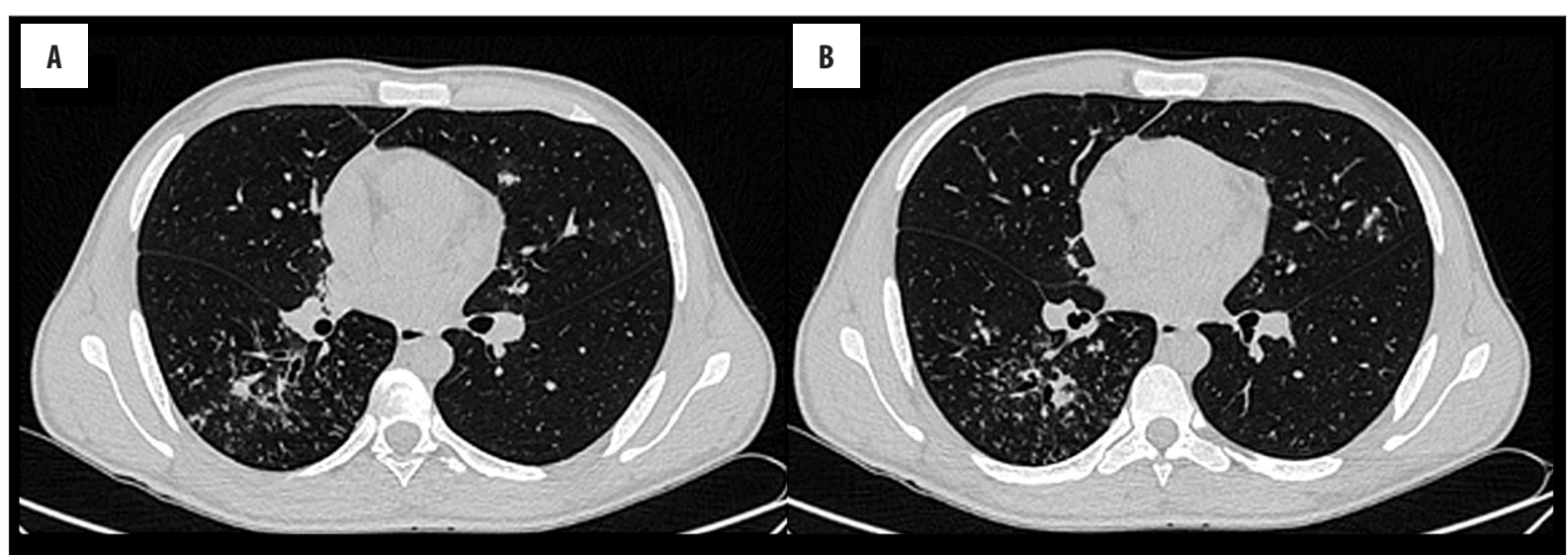

Figure 2. (A, B) Axial MD HRCT (pulmonary window) showing localized bronchiectatic changes with centrilobular nodules arranged in tree in bud configuration in the apical segment of the right lower lobe associated with centrilobular nodules arranged in tree in bud configuration in the inferior segment of the lingua in a 45 years old male complaining of cough, expectoration and weight loss.

cause an important medical problem in daily medical practice that is difficult to analyze [8].

Clinicians have some difficulties about whether antituberculous therapy should be initiated for these patients. Prompt initiation of anti-tuberculous therapy for pulmonary TB is an important issue both because of its benefits for the patient and for control of the disease [9].

It may not be appropriate to use the same criteria for cases of both smear-positive and smear-negative pulmonary TB to predict the risk in the latter group patients since smearnegative patients have lower mycobacterium burden [10].

In our study, we combined the clinical features together with the HRCT findings to differentiate smear-negative and culture- negative PTB from smear-negative and culturepositive disease. Cough was the most common symptom reported (90\% of the cases). Nakashi et al., [3] also reported that cough was seen in $83 \%$ of patients as the most common symptom in smear-negative PTB [3]. This was on the contrary of a study developed to validate a clinical-radiological score to assess the probability of pulmonary TB in patients suspected of smear negative pulmonary TB, productive cough was less frequent in smear negative pulmonary TB patients [11]. This was in agreement with a multivariate model, having no cough and no radiographic pattern typical of TB were the characteristics associated with a diagnosis of smear negative pulmonary TB [12].

Chest pain is the second presenting complaint, related to pleural involvement and considered a symptom for PTB. Tozkoparan et al., reported chest pain is the most common symptom (38\%) for smear negative PTB and confirmed the high existence of chest pain in active smear-negative PTB [10].

Other related symptoms in our patients were expectoration, hemoptysis, fever and weight loss. Our results showed that smear-negative culture-positive PTB cannot be discriminated from smear-negative and culture-negative PTB based on clinical symptoms and laboratory parameters. The presence of a dry cough, a high respiratory rate, a low eosinophil count, a mixed type of anemia and presence of a cavity were found to be predictive of smear negative but culture positive PTB [13].

The value of CT chest in diagnosing pulmonary TB was studied by many authors: Lee KS and colleagues in 1996 [14] studied the utility of CT in the evaluation of TB among patients without AIDS, they succeeded to predict presence of TB in 133 out of 146 patients proved to have 


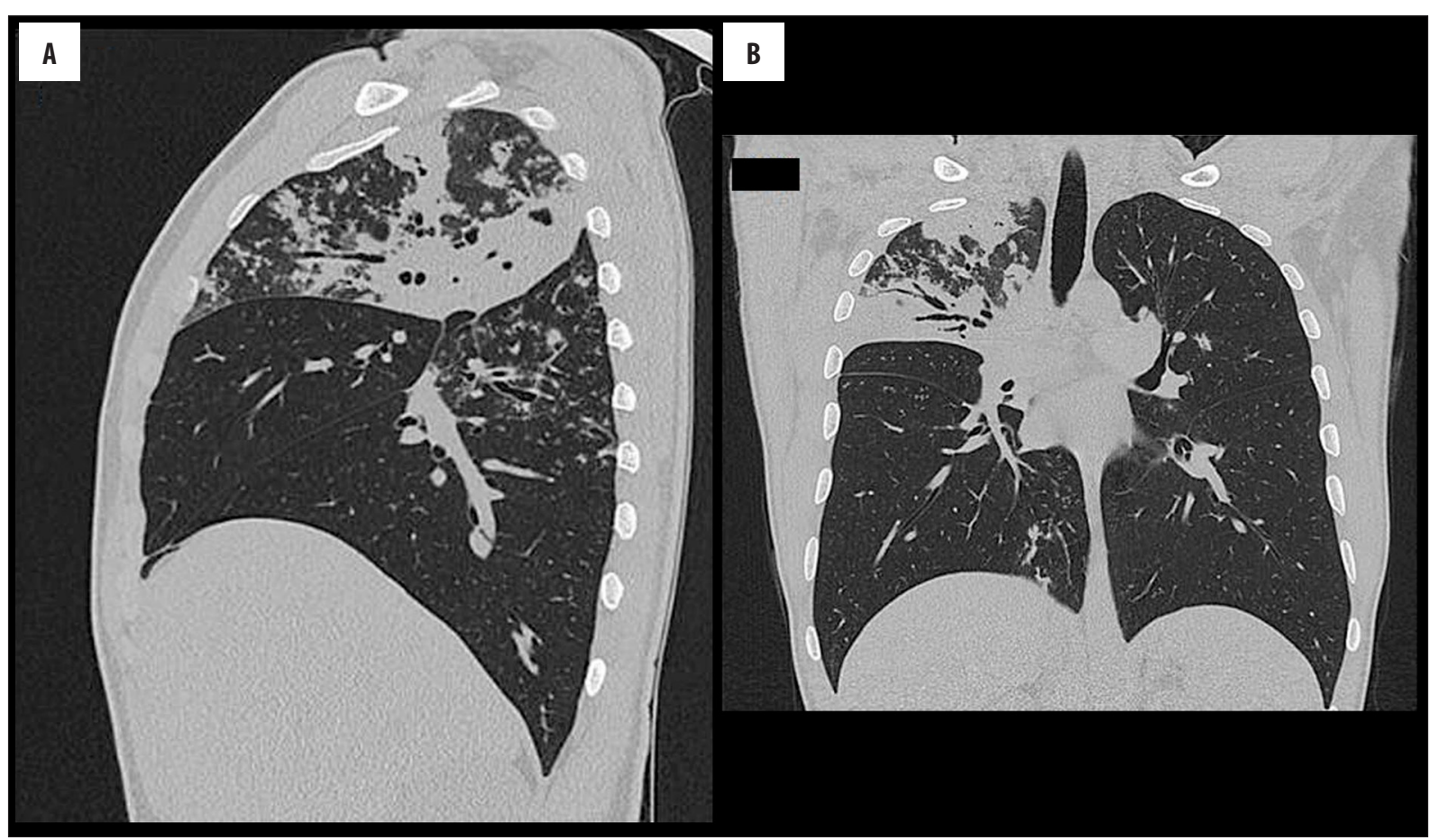

Figure 3. Sagittal (A) and coronal (B) MD HRCT (pulmonary window) showing right upper lobar consolidated area with air bronchogram associated with right upper lobar centrilobular nodules arranged in tree in bud configuration associated with localized bronchiectatic changes in the apical segment of the right upper lobe as well as the apical segment of the right lower lobe in a 30 years old male complaining of cough, chest pain and hemoptysis.

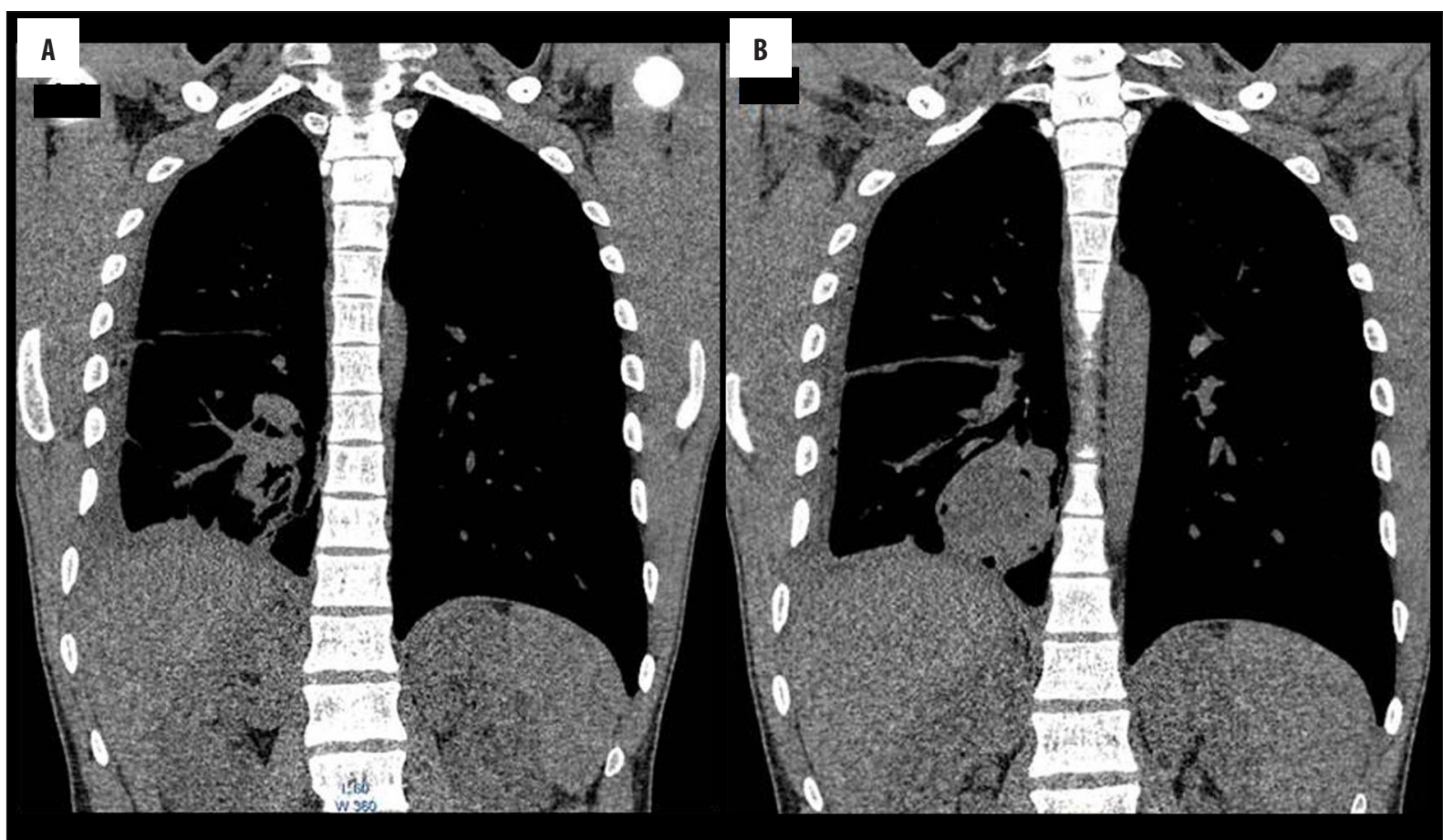

Figure 4. (A, B) Coronal MD HRCT (mediastinal window) showing right sided lamellar pleural effusion and consolidated area with air bronchogram inside seen in the medial segment of the right lower lobe in a 38 years old male patient complaining of fever, weight loss and night sweating. 


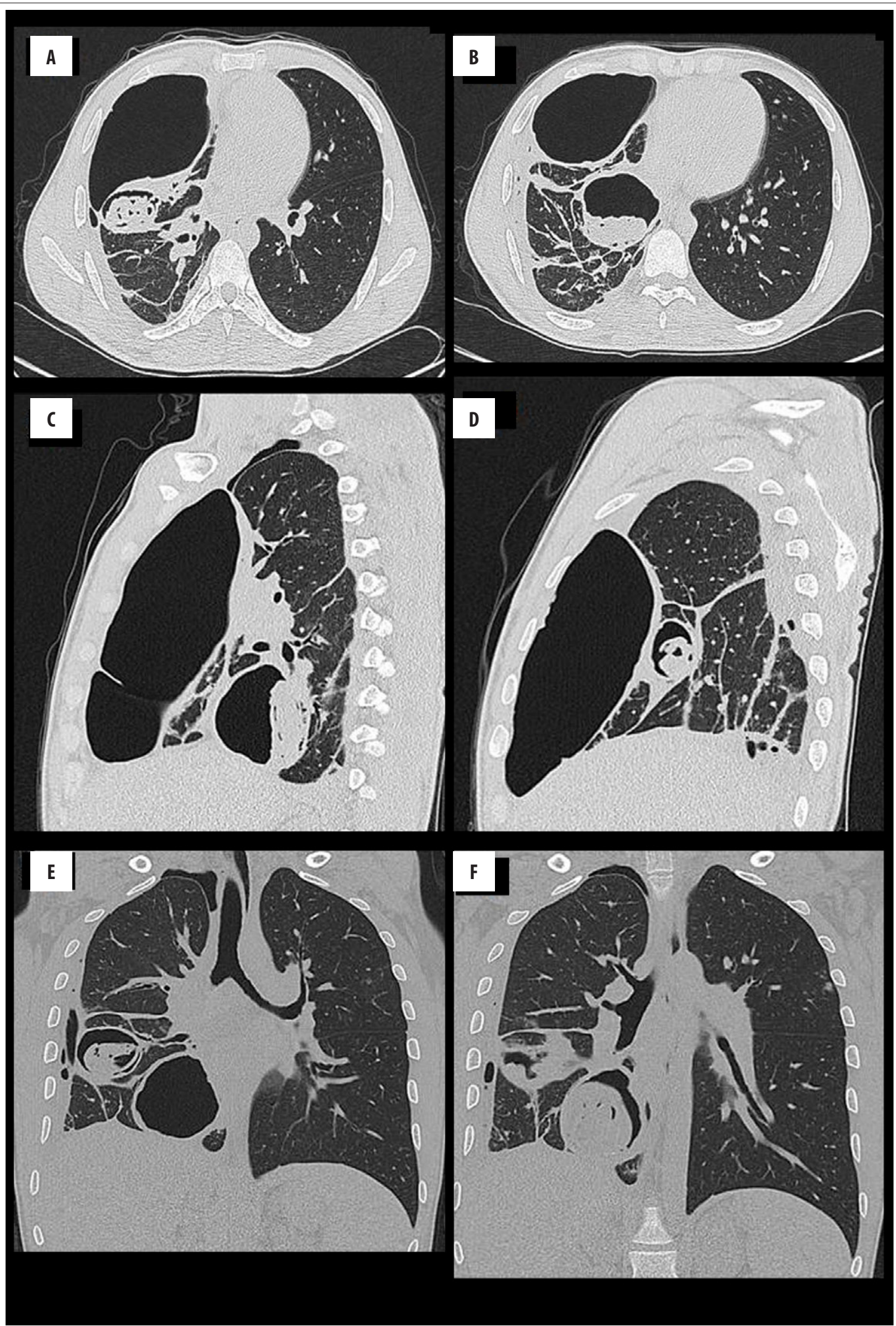

Figure 5. Axial (A, B), Sagittal (C, D) and coronal (E, F) MD HRCT (pulmonary window) showing large anterior air filled cavity occupying most of the right upper lobe extending from the lung apex down to the right diaphragmatic copula associated with two thickened wall cavities, each harboring solid content (mycetoma formation) at the apical segment of the right lower lobe with air crescent sign in a 62 years old male presented with cough, dyspnea, expectoration and weight loss. 
TB and to exclude TB in 32 out of 42 patients proved to have other diseases, they concluded that CT can be helpful in the diagnosis of pulmonary tuberculosis in most cases. On the basis of CT findings, distinction of active from inactive disease can be made in most cases. Lee SW and colleagues in 2010 [15] studied the use of CT in investigation of TB out break and with the use of CT they could diagnose active TB in nine patients who had normal chest X-ray and they concluded that adding CT to routine investigation of TB outbreak may be helpful in differentiating active TB from Latent TB infection.

HRCT findings in patients with active pulmonary $\mathrm{TB}$ include; micronodules, tree in bud appearance, airspace consolidation, nodules, ground glass opacities and cavities [16].

Matsuoka et al., [17] investigate the relationship between computed tomography (CT) findings in patients with active pulmonary tuberculosis (PTB) and number of the acid-fastbacilli (AFB) on sputum smears and they found that the frequency of micronodules and nodules did not significantly differ among the smear positive and smear negative groups. In contrast, the frequency of consolidation and cavitation increased with the number of AFB. In another study by Kosaka and his colleagues in 2005 [6] they found that air space consolidation, cavitation and ground glass opacities occurred significantly more frequently in the smear positive than in the smear negative active PTB patients while the frequency of centrilobular nodules (micronodules) did not differ between the two groups.

Tozkoparan et al., [10] found that HRCT had good diagnostic value in detecting activity of smear negative pulmonary tuberculosis. In their series the sensitivity, specificity, positive predictive value and accuracy of HRCT in detecting disease activity were $88 \%, 88 \%, 92 \%$ and $88 \%$, respectively. On the other hand in the study of Lee et al., [16] the sensitivity, specificity, positive predictive value and positive likelihood ratio of HRCT in the diagnosis of smear negative pulmonary tuberculosis were $80 \%, 70 \%, 71 \%$ and 2.71 . They concluded that HRCT alone had relatively good sensitivity but the low positive predictive value hampered the decision of starting anti-TB medication. However in the previous two studies, the HRCT diagnosis of active pulmonary TB was by consensus. Nakanishi and his colleagues in 2010 [3] investigated whether or not HRCT can predict risk for sputum smear-negative pulmonary TB. They ranked the patients from 1 to 3 based on a mixture of the HRCT findings that were significantly correlated with increased risk of pulmonary TB. Those comprised; large nodules, tree in bud appearance, lobular consolidation and presence of main lesion in S1, S2 and S6 segments). They found that this ranking was reliable enough to predict the risk of pulmonary TB with good reproducibility. Their results included the following: Rank 3 had sensitivity, specificity and positive likelihood ratio of $40 \%, 97 \%$ and 13.3 while rank 2 had 85\%, 74\% and 3.27 as sensitivity, specificity and positive likelihood ratio respectively. These results are confirmed by the results of our study. In this study the sensitivity, specificity and positive likelihood ratio of rank 3 were $45 \%, 90 \%$ and 11.5 respectively while rank 2 the results were $85 \%, 72 \%$ and 3 respectively. Our results are also keeping with the study done by Shaarrawy et al. [4] where the sensitivity, specificity and positive likelihood ratio of rank 3 were $48 \%, 91 \%$ and 12.5 respectively and in rank 2 there results were $79 \%, 70 \%$ and 3.1 respectively

The main role of HRCT for diagnosing pulmonary TB is the selection of probable or highly suspected pulmonary TB with pulmonary infiltrates of unknown origin and with negative sputum smears [3].

In the present study 12 out of the 51 ranked at HRCT as rank II and 40 out of the 44 rank III patients had final diagnosis of active pulmonary TB.

\section{Conclusions}

To conclude, for prediction of high risk patients among those with suspicion of having active pulmonary TB whose sputum smears are negative, HRCT findings are more or less of great value. Beside that, these findings can be applied to choose candidate patients for further laboratory tests or bronchoscopy.

\section{Conflict of interest}

The authors declared no conflict of interest.

\section{References:}

1. Caliskan T, Ozkisa T, Aribal S et al: High resolution computed tomography findings in smear-negative pulmonary tuberculosis patients according to their culture status. J Thorac Dis, 2014; 6(6): $706-12$

2. Global Tuberculosis Report, WHO (2012): Available online: $h t t p: / /$ www.who.int/tb/publications/global_report/gtbr12_main.pdf

3. Nakanishi M, Demura Y, Ameshima S et al: Utility of high-resolution computed tomography for predicting risk of sputum smear-negative pulmonary tuberculosis. Eur J Radiol, 2010; 73: 545-50

4. Shaarrawy H, Zeidan M, Nasr A et al: Assessment of the role of high resolution computed tomography in the diagnosis of suspected sputum smear negative active pulmonary TB. Egypt $J$ Chest Dis Tuberc, 2013; 62: 263-68

5. Lee HM, Shin JW, Kim JY et al: HRCT and whole-blood Interferon- $\gamma$ assay for the rapid diagnosis of smear negative pulmonary tuberculosis. Respiration, 2010; 79: 454-60
6. Kosaka N, Sakai T, Uematsu H et al: Specific high resolution computed tomography findings associated with sputum smear positive pulmonary tuberculosis. J Comput Assist Tomogr, 2005; 29: 801-4

7. Yeh JJ, Yu JK, Teng WB et al: High-resolution CT for indentify patients with smear positive active pulmonary tuberculosis. Eur J Radiol, 2012; 81: 195-201

8. Mello FC, Bastos LG, Soares SL et al: Predicting smear negative pulmonary tuberculosis with classification trees and logistic regression: A cross-sectional study. BMC Public Health, 2006; 6: 43

9. Affolabi D, Akpona R, Odoun $\mathrm{M}$ et al: Smear-negative, culturepositive pulmonary tuberculosis among patients with chronic cough in Cotonou, Benin. Int J Tuberc Lung Dis, 2011; 15: 67-70

10. Tozkoparan E, Deniz O, Ciftci F et al: The roles of HRCT and clinical parameters in assessing activity of suspected smear negative pulmonary tuberculosis. Arch Med Res, 2005; 36: 166-70

11. Soto A, Solari L, Diaz J et al: Validation of a clinical-radiographic score to assess the probability of pulmonary tuberculosis in suspect patients with negative sputum smears. PLoS One, 2011; 6(4): el8486 
12. Campos LC, Vieira Rocha MV, Cunha Willers DM et al: Characteristics of Patients with Smear-Negative Pulmonary Tuberculosis (TB) in a Region with High TB and HIV Prevalence. PLoS One, 2016; 11(1): e0147933

13. Swail HF, Mugusi FM, Mbwambo JK: Sputum smear negative pulmonary tuberculosis: sensitivity and specificity of diagnostic algorithm. 2011; http://www.biomedcentral.com/1756-0500/4/475

14. Lee KS, Hwang JW, Chung MP et al: Utility of CT in the evaluation of pulmonary tuberculosis in patients without AIDS. Chest, 1996; 110: 977-84
15. Lee SW, Jang YS, Park CM et al: The role of chest CT scanning in TB outbreak investigation. Chest, 2010; 137(5): 1057-64

16. Lee JJ, Chong PY, Lin CB et al: High resolution CT in patients with pulmonary tuberculosis: characteristic findings before and after antituberculous therapy. Eur J Radiol, 2008; 67: 100-4

17. Matsuoka S, Uchiyama K, Shima H et al: Relationship between CT findings of pulmonary tuberculosis and the number of acid-fast bacilli on sputum smears. Clin Imaging, 2004; 28: 119-23 